\begin{tabular}{|l|l|}
\hline Title: & $\begin{array}{l}\text { Grid Side Inverter Control Scheme for Robust Fault Ride Through } \\
\text { with Enhanced Overload Capabilities }\end{array}$ \\
\hline Authors: & Bernd Bohnet, Thomas Jambor, Philipp Gutknecht, Michael Braun \\
\hline Institute: & $\begin{array}{l}\text { Karlsruhe Institute of Technology (KIT) } \\
\text { Elektrotechnisches Institut (ETI) }\end{array}$ \\
\hline Type: & Conference Proceedings \\
\hline Published at: & $\begin{array}{l}\text { IECON } 2016 \text { - 42nd Annual Conference of the IEEE Industrial Electronics Society } \\
\text { Florence, Italy, 23-26 Oct. } 2016 \\
\text { Publisher: IEEE } \\
\text { ISBN: } 978-1-5090-3474-1 \\
\text { Pages: } 2307-2312\end{array}$ \\
\hline Hyperlinks: & DOI: $10.1109 /$ IECON.2016.7793273 \\
\hline
\end{tabular}

(C) 2016 IEEE. Personal use of this material is permitted. Permission from IEEE must be obtained for all other uses, in any current or future media, including reprinting/republishing this material for advertising or promotional purposes, creating new collective works, for resale or redistribution to servers or lists, or reuse of any copyrighted component of this work in other works. 


\title{
Grid Side Inverter Control Scheme for Robust Fault Ride Through with Enhanced Overload Capabilities
}

\author{
Bernd Bohnet, Thomas Jambor, Philipp Gutknecht and Michael Braun \\ Karlsruhe Institute of Technology (KIT) \\ Elektrotechnisches Institut (ETI) - Electrical Drives and Power Electronics \\ 76131 Karlsruhe, Germany \\ bernd.bohnet@kit.edu
}

\begin{abstract}
In this paper a predictive control scheme for current injection into three phase grids with power converters is presented. The proposed control scheme shows an excellent current quality even under adverse grid conditions like unsymmetrical grid faults.

By usage of iron powder inductors as filter elements for the grid current a very high overload capability of the inverter can be achieved without oversizing these filter elements. The performance of the proposed control is experimentally verified with a two-level three-phase active front end.
\end{abstract}

\section{INTRODUCTION}

Today the largest share of energy is still produced by means of large scale power plants, but energy production by distributed energy resources has reached a considerable amount. This share will even increase in the future, especially with the environmental targets addressed by the German federal government. According to [1], the amount of energy produced by regenerative power plants is planned to be $80 \%$ in the year 2050. In the same effort the last nuclear power plant will be shut down until 2021.

These values show that the power grid will undergo a major transformation in the next few years, from a centralized to a mostly distributed energy production. Most of the distributed energy resources are interfaced to the grid by means of power electronic converters. In contrast to these converters the synchronous machines used in large scale power plants show an inherent robust behaviour against grid faults like short circuits, voltage sags or frequency deviations. Additionally, classical synchronous generators feature a high current overload capability during such faults, which helps tripping protection systems and increase grid stability.

In future grids dominated by power electronics, it is important that this robustness - which is realised by power plants today - is implemented in a distributed manner. One important point therefore is maintaining of current control even under severe conditions - like grid faults - to support the grid [2]. Therefore many different control approaches were presented, for example [3]-[5].

However, inverters are normally limited to a maximum current near their nominal current rating. Since fault events, which require excessive currents for grid support, are rare [6], it is undesirable to increase this nominal rating. Instead, overloading capabilities of the inverter system should be used.
The power electronic device itself could be designed to enable a high current overload, especially with emerging SiC devices [6], [7]. Another limiting factor is the grid interfacing filter element, which normally is imposed to saturation effects under excessive currents, so controllability of the inverter is challenging and system perturbations increase.

Addressing these drawbacks, a predictive current control scheme is presented, which maintains high current quality and controllability even under severe grid-faults. It is shown, that by the usage of iron powder inductances as grid interfacing filter elements, high overload currents of up to $250 \%$ of the nominal value are possible. A disadvantage is, that iron powder inductors do not show a linear relationship between flux linkage $\Psi$ and current $i$, so predictive control approaches which assume a linear relationship - like presented in [8] or [9] - are likely to result in poor control quality.

In [10] a predictive current controller is suggested which maintains control over the currents in synchronous machines even under strong saturation effects. A rather similar control loop is implemented and adapted for the purpose of grid current injection.

In Section II the hardware setup is described and the model for control implementation is derived. In Section III the predictive control algorithm is presented. Section IV explains the prototype testbed and Section V validates the model using measurement results.

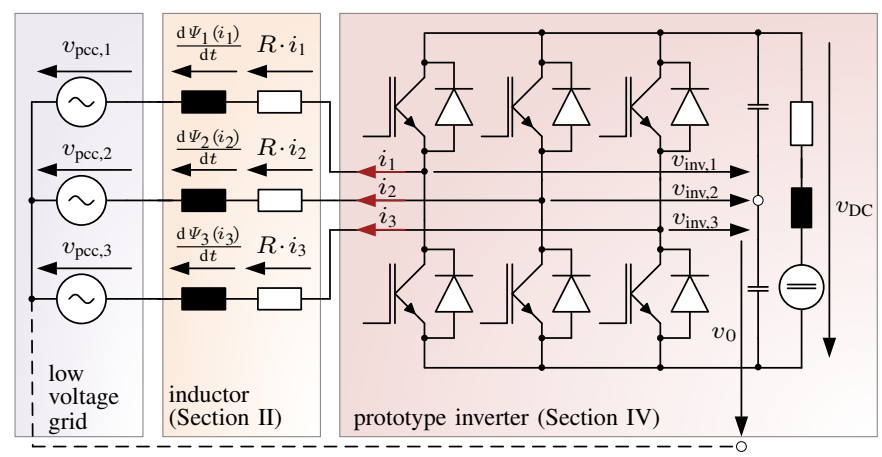

Figure 1. Overview of the investigated system for modelling and control implementation 


\section{HARDWARE SETUP}

Figure 1 gives an overview of the investigated system. It consists of a standard two-level three-phase bridge, interfaced to the grid by a filter consisting of three uncoupled inductors.

In other applications these inductors are often coupled to economize design, but single inductors are advantageous in some cases because zero sequence currents through parasitic elements can be reduced and emc considerations can be met. Despite the missing magnetic coupling of the inductor, this configuration can be used without loss of generality, as is shown in Section II-B.

\section{A. Choice of the magnetic material for the filter elements}

As stated in Section I, besides the layout of the power electronic switches itself - which is not within the scope of this paper - the grid interfacing filter elements have to be reviewed to evaluate possible current overload capability of the whole converter system.

Materials for the filter inductors cores can be divided into the three main groups:

- laminated cores

- ferrite cores

- iron powder cores

Whereas all materials have different characteristics, for assessing overload capability especially the dependence of flux linkage $\Psi$ and current $i$ and hence with

$$
L_{\mathrm{diff}}=\frac{\mathrm{d} \Psi}{\mathrm{d} i}
$$

the evolution of the differential inductance $L_{\text {diff }}$ are important If the decrease in differential inductance is too steep, this can lead to undue system perturbations, which should be avoided for power quality reasons.

To rate this behaviour, an exemplary measurement of three different inductors was carried out. In Figure 2 the characteristics of the differential inductances depending on the currents are plotted.

To simplify comparison, the measurements are normalized regarding the nominal inductance values and the currents at which saturation starts. For the iron powder inductor the values are normalized to the design nominal values.

It is obvious that inductors built with laminated or ferrite cores show a region of nearly constant inductance, whereas

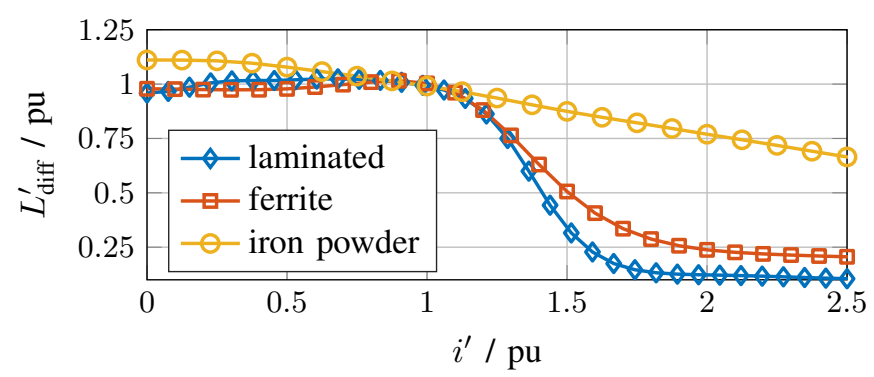

Figure 2. Measurements of the differential inductance of different core materials, normalized to rated inductance and saturating current. beyond this point they show excessive saturation characteristics. To enable high overload currents with these core types, the core has to be chosen bigger than needed, otherwise only rather small overload factors are possible.

In contrast, the iron powder inductor features a very soft characteristic, where no abrupt change in inductance is present. This enables a high overload capability without sacrificing current quality impermissibly during overload operation. $L_{\text {diff }}$ varies over the whole current range, meaning there is no simple linear relationship between flux linkage and current.

\section{B. Modelling}

To gain a mathematical expression for the plant, first the equations for the three-phase system are given:

$$
\begin{aligned}
\left(\begin{array}{l}
v_{\text {inv }, 1} \\
v_{\text {inv }, 2} \\
v_{\text {inv }, 3}
\end{array}\right)+\left(\begin{array}{l}
v_{0} \\
v_{0} \\
v_{0}
\end{array}\right)= & \frac{\mathrm{d}}{\mathrm{d} t}\left(\begin{array}{l}
\Psi_{1}\left(i_{1}\right) \\
\Psi_{2}\left(i_{2}\right) \\
\Psi_{3}\left(i_{3}\right)
\end{array}\right)+ \\
& \left(\begin{array}{rr}
R & \\
R \\
R
\end{array}\right)\left(\begin{array}{c}
i_{1} \\
i_{2} \\
i_{3}
\end{array}\right)+\left(\begin{array}{c}
v_{\mathrm{pcc}, 1} \\
v_{\mathrm{pcc}, 2} \\
v_{\mathrm{pcc}, 3}
\end{array}\right)
\end{aligned}
$$

Here $v_{\text {inv }, x}, v_{\text {pcc }, x}, \Psi_{x}$ and $i_{x}$ with $x \in\{1,2,3\}$ denote the respective inverter output voltage, grid voltage, flux linkage and inverter output current. $v_{0}$ denotes the zero sequence voltage measured from the split dc-link potential with respect to the neutral line of the three-phase grid. The resistance value $R$ is assumed to be equal for all inductors.

Equation (2) is transformed using the Clark transformation:

$$
\vec{x}_{\alpha \beta 0}=\left(\begin{array}{l}
x_{\alpha} \\
x_{\beta} \\
x_{0}
\end{array}\right)=\frac{2}{3}\left(\begin{array}{ccc}
1 & -\frac{1}{2} & -\frac{1}{2} \\
0 & \frac{\sqrt{3}}{2} & -\frac{\sqrt{3}}{2} \\
\frac{1}{2} & \frac{1}{2} & \frac{1}{2}
\end{array}\right)\left(\begin{array}{l}
x_{1} \\
x_{2} \\
x_{3}
\end{array}\right)
$$

This transformation rule applied on equation 2 yields

$$
\begin{aligned}
& \left(\begin{array}{c}
v_{\text {inv }, \alpha} \\
v_{\text {inv }, \beta} \\
v_{\text {inv }, 0}
\end{array}\right)+\left(\begin{array}{c}
0 \\
0 \\
v_{0}
\end{array}\right)=\frac{\mathrm{d}}{\mathrm{d} t}\left(\begin{array}{l}
\Psi_{\alpha} \\
\Psi_{\beta} \\
\Psi_{0}
\end{array}\right)+ \\
& \left(\begin{array}{lll}
R & & \\
& R & \\
& & R
\end{array}\right)\left(\begin{array}{l}
i_{\alpha} \\
i_{\beta} \\
i_{0}
\end{array}\right)+\left(\begin{array}{l}
v_{\mathrm{pcc}, \alpha} \\
v_{\mathrm{pcc}, \beta} \\
v_{\mathrm{pcc}, 0}
\end{array}\right)
\end{aligned}
$$

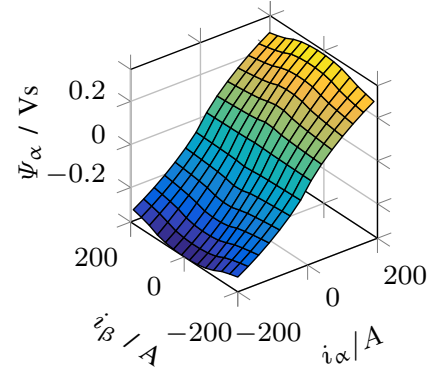

(a) Flux linkage $\Psi_{\alpha}$

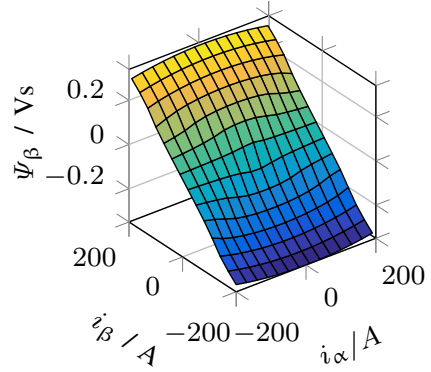

(b) Flux linkage $\Psi_{\beta}$
Figure 3. Plots of the resulting flux linkages after Clark transformation 


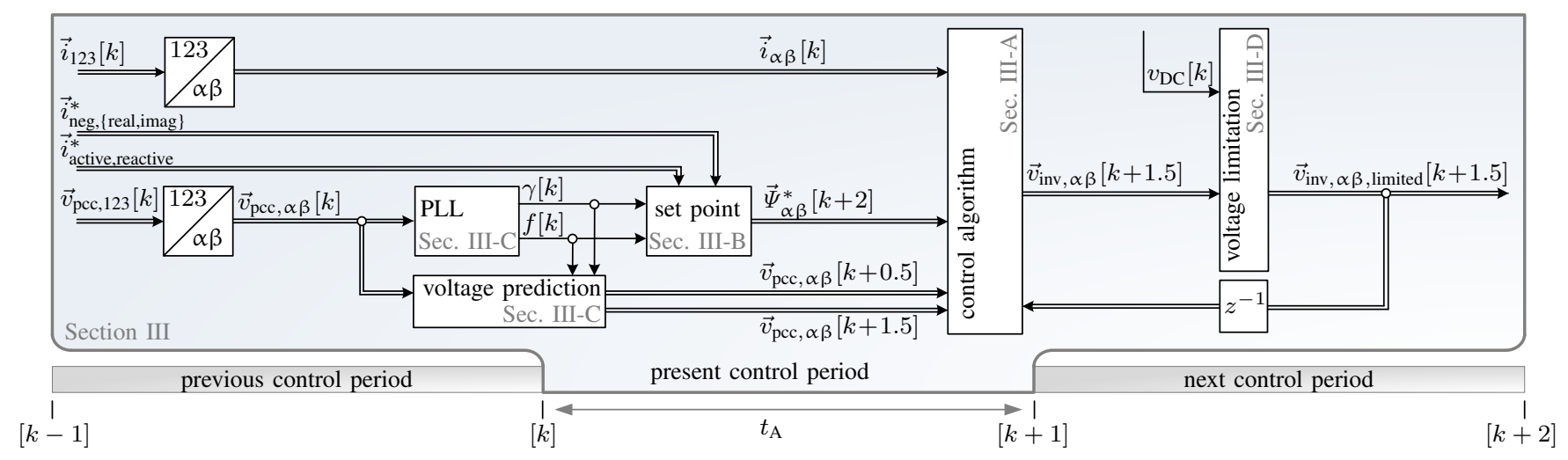

Figure 4. Implemented predictive current control scheme

Because the neutral line is not connected, a zero sequence current can not exist $\left(i_{0}=0\right)$ and the last line of Equation (4) can safely be omitted. This finally yields

$$
\left(\begin{array}{l}
v_{\text {inv, } \alpha} \\
v_{\text {inv }, \beta}
\end{array}\right)=\underbrace{\frac{\mathrm{d}}{\mathrm{d} t}\left(\begin{array}{l}
\Psi_{\alpha} \\
\Psi_{\beta}
\end{array}\right)}_{\begin{array}{c}
\text { inductive } \\
\text { voltage drop }
\end{array}}+\underbrace{\left(\begin{array}{c}
R \\
R
\end{array}\right)\left(\begin{array}{l}
i_{\alpha} \\
i_{\beta}
\end{array}\right)}_{\begin{array}{c}
\text { resistive } \\
\text { voltage drop }
\end{array}}+\left(\begin{array}{l}
v_{\mathrm{pcc}, \alpha} \\
v_{\mathrm{pcc}, \beta}
\end{array}\right)
$$

Equation (5) is given here in more compact matrix notation for later usage:

$$
\vec{v}_{\text {inv }, \alpha \beta}=\frac{\mathrm{d}}{\mathrm{d} t} \vec{\Psi}_{\alpha \beta}+\boldsymbol{R}_{\alpha \beta} \cdot \vec{i}_{\alpha \beta}+\vec{v}_{\mathrm{pcc}, \alpha \beta}
$$

The result of the clark-transformation for the vector of flux linkage is

$\vec{\Psi}_{\alpha \beta}=\left(\begin{array}{c}\Psi_{\alpha}\left(i_{\alpha}, i_{\beta}\right) \\ \Psi_{\beta}\left(i_{\alpha}, i_{\beta}\right)\end{array}\right)=\frac{2}{3}\left(\begin{array}{c}\Psi_{1}\left(i_{1}\right)-\frac{1}{2} \Psi_{2}\left(i_{2}\right)-\frac{1}{2} \Psi_{3}\left(i_{3}\right) \\ \frac{\sqrt{3}}{2} \Psi_{2}\left(i_{2}\right)-\frac{\sqrt{3}}{2} \Psi_{3}\left(i_{3}\right)\end{array}\right)$

Regarding equation 7 it is apparent that the resulting flux linkages $\Psi_{\alpha}$ and $\Psi_{\beta}$ are depending on the currents $i_{1}, i_{2}$ and $i_{3}$, so they can be described as functions of $i_{\alpha}$ and $i_{\beta}$.

With Equation (7), flux linkages $\Psi_{\alpha}$ and $\Psi_{\beta}$ can be calculated using measurements of a single inductor. The results are given in Figure 3 showing the effects of the current dependent inductance.

If three-phase inductors are used the derived system equations remain valid. In this case only the flux maps must be adapted according to the additional magnetic coupling.

\section{Control Scheme}

In Section II-B the model of the control plant was derived. Figure 4 shows a block diagram of the implemented control system, which is explained in the following sections.

Because of the non linear relationship between flux linkage $\vec{\Psi}_{\alpha \beta}$ and current $\vec{i}_{\alpha \beta}$, first the control of the flux linkage values is shown in Section III-A, since this remains a linear problem. For current set point generation and mapping between flux linkage values and current see Section III-B.

\section{A. Predictive Control}

For implementation of the control loop on a digital signal processing system, Equation (6) is given in discrete time:

$$
\begin{aligned}
\vec{v}_{\text {inv }, \alpha \beta}[k+0.5]=\frac{\vec{\Psi}_{\alpha \beta}[k+1]-\vec{\Psi}_{\alpha \beta}[k]}{t_{\mathrm{A}}}+ & \boldsymbol{R}_{\alpha \beta} \cdot \vec{i}_{\alpha \beta}[k]+\vec{v}_{\mathrm{pcc}, \alpha \beta}[k+0.5]
\end{aligned}
$$

Sample instant $[k]$ denotes measurements, which are taken at the begin of the present control period (see also Figure 4). Subsequently instant $[k+1]$ denotes the values to the begin of the next control period. $t_{\mathrm{A}}$ corresponds to the duration of one control period.

In contrast the voltage values in Equation (8) are given to sample instants of $[k+0.5]$. This means, that for the necessary voltage vectors those values are regarded, which are effective for the respective control period. The grid voltage prediction method to gain these values is described in Section III-C.

The voltage $v_{\text {inv, } \alpha \beta}[k+0.5]$ obviously corresponds to that voltage, which is realized by the modulation process of the power converter.

Because of the switching nature of the power converter, a dead time of one switching period is inevitable to calculate the control scheme. The flux evolution during this dead time is calculated by means of Equation (8) to

$$
\begin{aligned}
\vec{\Psi}_{\alpha \beta}[k+1]=\left(\vec{v}_{\mathrm{inv}, \alpha \beta}[k+0.5]-\boldsymbol{R}_{\alpha \beta} \cdot \vec{i}_{\alpha \beta}[k]-\right. \\
\left.\vec{v}_{\mathrm{pcc}, \alpha \beta}[k+0.5]\right) \cdot t_{\mathrm{A}}+\vec{\Psi}_{\alpha \beta}[k]
\end{aligned}
$$

Based on this prediction, the necessary voltage to reach the reference flux linkage is then calculated with Equation (8) to:

$$
\begin{aligned}
\vec{v}_{\mathrm{inv}, \alpha \beta}[k+1.5] & =\frac{\vec{\Psi}_{\alpha \beta}^{*}[k+2]-\vec{\Psi}_{\alpha \beta}[k+1]}{t_{\mathrm{A}}}+ \\
& \boldsymbol{R}_{\alpha \beta} \cdot \vec{i}_{\alpha \beta}[k+1]+\vec{v}_{\mathrm{pcc}, \alpha \beta}[k+1.5]
\end{aligned}
$$

$\vec{\Psi}_{\alpha \beta}^{*}[k+2]$ denotes the flux linkage reference values (Section III-B)

Problems arise regarding the value for $\vec{i}_{\alpha \beta}[k+1]$. To determine this value there are principally two options: 
1) inversion of $\vec{\Psi}_{\alpha \beta}\left(i_{\alpha \beta}\right): \vec{i}_{\alpha \beta}[k+1]=f\left(\vec{\Psi}_{\alpha \beta}\right)$

2) usage of $i_{\alpha \beta}[k]: \vec{i}_{\alpha \beta}[k+1] \approx \vec{i}_{\alpha \beta}[k]$

Option one is exact, but also most challenging, regarding computational power and storage. The value for $\vec{i}_{\alpha \beta}[k+1]$ is derived by inversion of the flux maps. Since this is a computational extensive procedure, the calculation can be done offline. The solutions can then be used as look up table.

To avoid storage or online calculation time option two can be chosen. Regarding the resistive voltage drop in contrast to the inductive voltage drop (see Equation (5)), the resistive one is rather small, due to desired small resistance values. By choosing the already measured value $i_{\alpha \beta}[k]$ the resulting error is sufficiently small. This approach is implemented in the control algorithm, since degradation of control quality was not noticed at the test bench.

\section{B. Set point generation}

The generation of set points can be split into two parts.

1) Current reference estimation: First off all the set point currents must be generated. In a simple form, they are derived by specification of active and reactive currents. Therefore the desired active and reactive currents are rotated with:

$$
\boldsymbol{A}_{\Theta}=\left(\begin{array}{cc}
\cos \Theta & -\sin \Theta \\
\sin \Theta & \cos \Theta
\end{array}\right)
$$

In this case the angle $\Theta$ becomes

$$
\Theta_{\mathrm{ref}}=\gamma[k+2]=\gamma[k]+\omega \cdot 2 \cdot t_{\mathrm{A}}
$$

where $\gamma[k]$ denotes the momentary angle of the positive sequence of the grid voltage (see Section III-C), so even under adverse grid conditions a sinusoidal, balanced current is injected. The angle increment $\omega \cdot 2 \cdot t_{\mathrm{A}}$, with $\omega=2 \cdot \pi \cdot f$ is added, to account for the necessary prediction due to the dead time. Also variations in grid frequency $f$ are taken into account.

Using these equations the corresponding positive sequence currents on the $\alpha \beta$ plane can be calculated to:

$$
\left(\begin{array}{c}
i_{\text {pos }, \alpha}^{*} \\
i_{\mathrm{pos}, \beta}^{*}
\end{array}\right)=\boldsymbol{A}_{\Theta, \text { ref }} \cdot\left(\begin{array}{c}
i_{\text {active }}^{*} \\
i_{\text {reactive }}^{*}
\end{array}\right)
$$

where $i_{\text {active }}^{*}$ and $i_{\text {reactive }}^{*}$ denote the active and reactive current references. Obviously the trajectory of the reference current can be freely chosen - within the possible dynamic range of the inverter - so even injection of negative sequence currents or higher current harmonics is possible. By addition of

$$
\left(\begin{array}{l}
i_{\text {neg, } \alpha}^{*} \\
i_{\text {neg, }, \beta}^{*}
\end{array}\right)=-\boldsymbol{A}_{\Theta, \text { ref }} \cdot\left(\begin{array}{c}
i_{\text {neg,real }}^{*} \\
i_{\text {neg,imag }}^{*}
\end{array}\right)
$$

to the current references calculated with Equation (13), negative sequence currents can be injected for enhanced ancillary services. $i_{\text {neg,real }}^{*}$ and $i_{\text {neg,imag }}^{*}$ denote the respective real and imaginary reference values for the negative sequence vector.
2) Flux linkage reference estimation: After calculation of reference currents, the reference flux linkages for the control loop have to be determined. Therefore one could use a look up table with the data presented in Figure 3. With sufficient computing power it is also possible to calculate the fluxlinkage reference values $\vec{\Psi}_{\alpha \beta}^{*}[k+2]$ with help of Equation (7) directly from measured flux linkages $\Psi(i)$ for the used inductors and the reference currents calculated by Equation (13) and Equation (14).

The latter approach was implemented since computational power was no limiting factor.

\section{Grid Voltage Prediction}

As shown in Equation (10) and Figure 4, the control scheme depends on the angle $\gamma$ of the positive sequence space vector, grid frequency $f$ as well as the grid voltages $\vec{v}_{\alpha \beta}[k+0.5]$ and $\vec{v}_{\alpha \beta}[k+1.5]$. All these values need to be calculated from the measured grid voltage $\vec{v}_{\mathrm{pcc}, \alpha \beta}[k]$.

1) Phase Locked Loop: The angle $\gamma$ and frequency $f$ are needed for reference value generation. Since even under adverse grid conditions these values must be known with high accuracy, a stable phase locked loop (PLL) scheme is necessary. [11] and [12] deliver an overview of different possible implementations for such robust PLL structures. In the context of this paper, a similar PLL-structure like presented in [13] is implemented, so the desired values can be derived even under sudden grid faults.

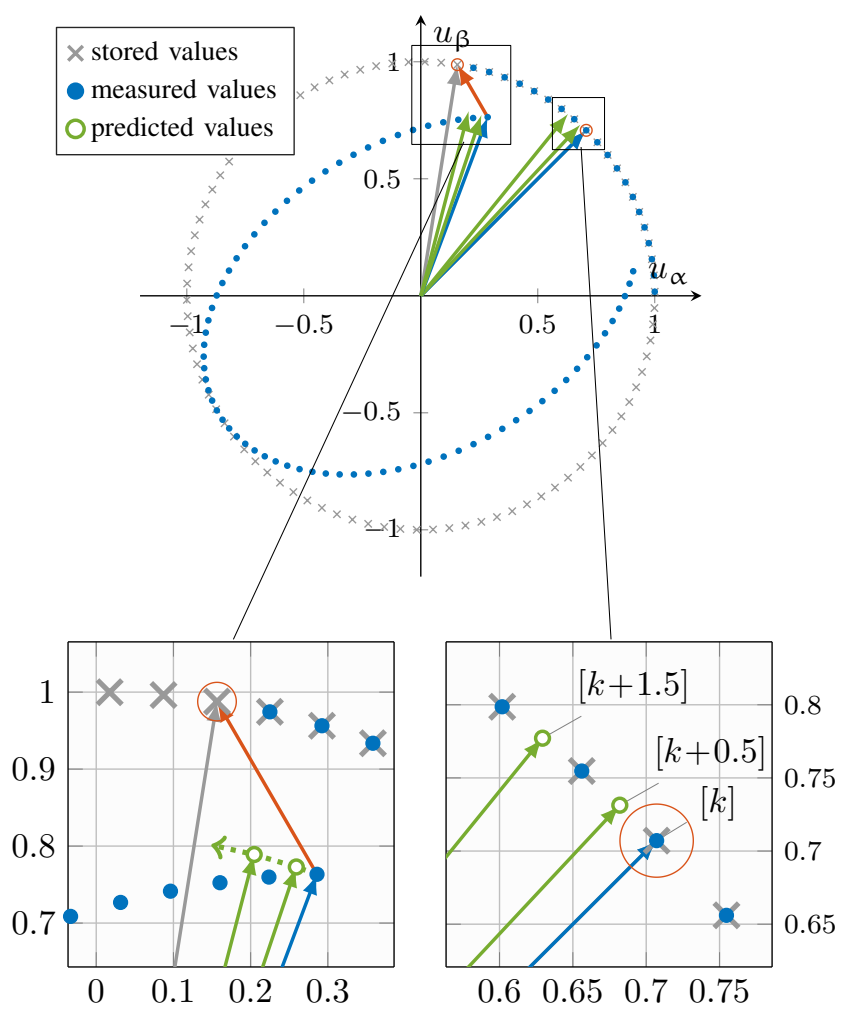

Figure 5. Voltage prediction during sudden grid faults 
2) Voltage Prediction: To enable a high prediction quality - even under non ideal conditions - a circular buffer is implemented for the generation of prediction values for $\vec{v}_{\alpha \beta}[k+0.5]$ and $\vec{v}_{\alpha \beta}[k+1.5]$. A similar buffer was used before in [14] to predict voltage errors for electrical machines.

In the bottom right cut out of Figure 5 the prediction procedure implemented for the presented control scheme is shown. Blue dots ( $\bullet$ denote the currently measured value at the instant $[k]$. The desired prediction values for the grid voltage vector to the instants $[k+0.5]$ and $[k+1.5]$ are denoted by green circles $(0)$. Since the trajectory of the grid voltage space vector is highly stationary (under normal conditions), it is feasible to store measured values in a circular buffer (values denoted by grey crosses $(x)$ ). The depth of this buffer is chosen to hold all measured values from the last grid voltage period. With help of these values, the prediction can be done with high accuracy by interpolation between corresponding values from the last grid period. This procedure even considers voltage harmonics and existing negative sequence voltages.

Disadvantageous is the slow reaction in case of sudden changes in grid voltage - like grid faults - since a full grid period is needed, to update all values in the circular buffer. Subsequently no meaningful prediction is possible during this time. To enable stable current control even during such faults, a second prediction algorithm is implemented, which is explained in the bottom left cut out of Figure 5. The currently measured voltage vector will simply be rotated with help of Equation (11). The angle $\Theta$ in this case is chosen to $\Theta_{[k+0.5]}=\omega \cdot 0.5 \cdot t_{\mathrm{A}}$ and $\Theta_{[k+1.5]}=\omega \cdot 1.5 \cdot t_{\mathrm{A}}$ respectively. Resulting errors due to this imperfect prediction stay sufficiently low, so a stable grid current injection is possible.

3) Fault detection: The stored values are also used to decide which algorithm is used for voltage prediction. For every measured value, the corresponding value from the last grid period is read from the buffer and compared. If the euclidean distance between these two vectors (denoted with a red arrow $(\longrightarrow)$ ) is too large (red circle in Figure 5), the second algorithm is used for the subsequent grid period. If after this time a new stationary grid voltage trajectory has established, the prediction algorithm is switched back to the circular buffer approach. This fault detection scheme can also be used to set new reference values during grid faults.

\section{Voltage limitation}

After the voltage vector $\vec{u}_{\text {inv }, \alpha \beta}[k+1.5]$ is calculated, it must be ensured that it can be realized. If this is not possible, due to a limited dc link voltage, the control error for the next control period has to be minimized. Even though the presented current control is implemented in $\alpha \beta$ coordinates, the output voltage limitation technique presented in [15] can be used for voltage limitation.

The limited voltage $\vec{u}_{\text {inv, } \alpha \beta \text {,limited }}[k+1.5]$ is delayed and fed back into the control algorithm for usage in Equation (9) during the next control period. Finally the resulting voltage vector is realized by the inverters modulation process.
Table I

KEY DATA OF THE USED PROTOTYPE SETUP

\begin{tabular}{lll}
\hline inverter & $\begin{array}{l}\text { switching frequency } \\
\text { dc-link voltage }\end{array}$ & $\begin{array}{l}8 \mathrm{kHz} \\
450 \mathrm{~V}\end{array}$ \\
\hline \multirow{2}{*}{ low voltage grid } & nominal line to line voltage & $125 \mathrm{~V}$ \\
& nominal grid frequency & $50 \mathrm{~Hz}$ \\
\hline \multirow{2}{*}{ inductance } & rated inductance & $2 \mathrm{mH}$ \\
& rated current & $45 \mathrm{~A}$ \\
\hline
\end{tabular}

\section{PRototype Setup}

\section{A. Prototype Hardware}

For the measurements an inverter consisting of three Infineon PrimePACK FF450R12IE4 modules was used. Due to their high current rating they are ideal for verification of the overload capabilities of the grid filter elements, because no inverter current limit would be reached. Necessary gate units are custom built to enable full flexibility for special control implementations. Further data regarding the laboratory prototype is given in Table I.

The power converter is integrated into a low voltage grid. Additionally one phase is connected through an autotransformer, which allows evaluation of asymmetric fault ride through conditions.

\section{B. Real Time Control System}

The control loop is implemented on a flexible real-time system consisting of a Digital-Signal-Processor from Texas Instruments, which enables the complete calculation in less than $125 \mu$ s to reach a switching frequency of $8 \mathrm{kHz}$. Additional hardware like analog to digital converters and field programmable gate arrays for clock generation are implemented as plug-in modules.

\section{Measurements}

\section{A. Fault Ride Through}

To investigate the fault-ride-through behaviour, a measurement with a sudden short circuit of $v_{\text {pcc, } 2}$ at the point of common coupling was carried out (Figure 6).

The inverters operating point before shorting was set to $i_{\text {active }}^{*}=\sqrt{2} \cdot 20 \mathrm{~A}$ and $i_{\text {reactive }}^{*}=0 \mathrm{~A}$. Right after detection of the short-circuit with the algorithm described in Section III-C, the current was automatically set to $i_{\text {active }}^{*}=0 \mathrm{~A}$ and $i_{\text {reactive }}^{*}=$ $-\sqrt{2} \cdot 120$ A. The implemented current control scheme shows good stability, even during rapidly changing grid voltages a large signal step is possible. Additionally the high overload capability of the iron powder inductors (Figure 2) is validated successfully. Regarding the nominal current rating, here an overload factor of circa $250 \%$ is demonstrated.

\section{B. Positive and Negative Sequence Injection}

Like stated in Section III-B, the reference current trajectory could be chosen freely. The measurement presented in Figure 7 demonstrates this possibility by injecting a negative sequence current into the grid. Additionally, the line voltage $v_{\mathrm{pcc}, 2}$ was lowered to approximately $60 \%$ of its nominal value. 

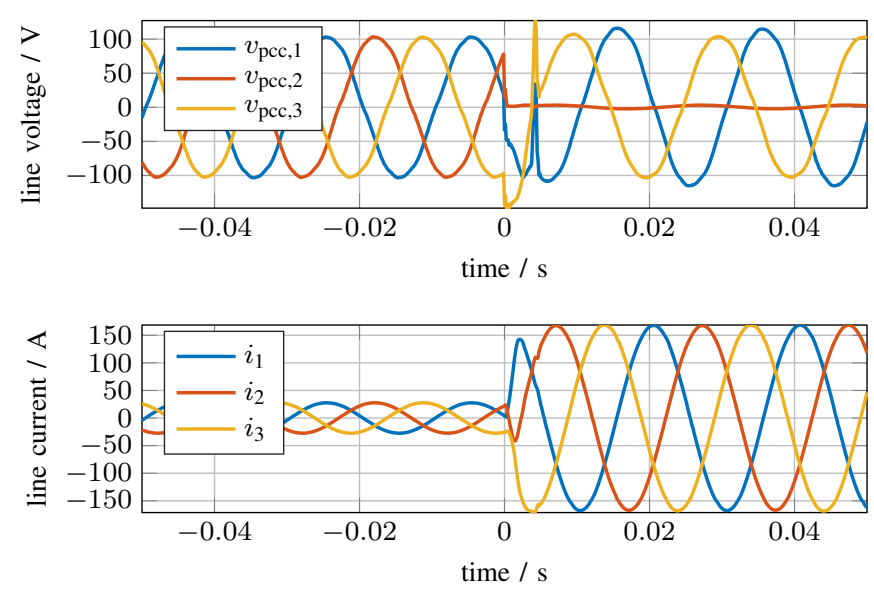

Figure 6. Fault ride through behaviour. Shown are the sampled values captured with the aforementioned control system.
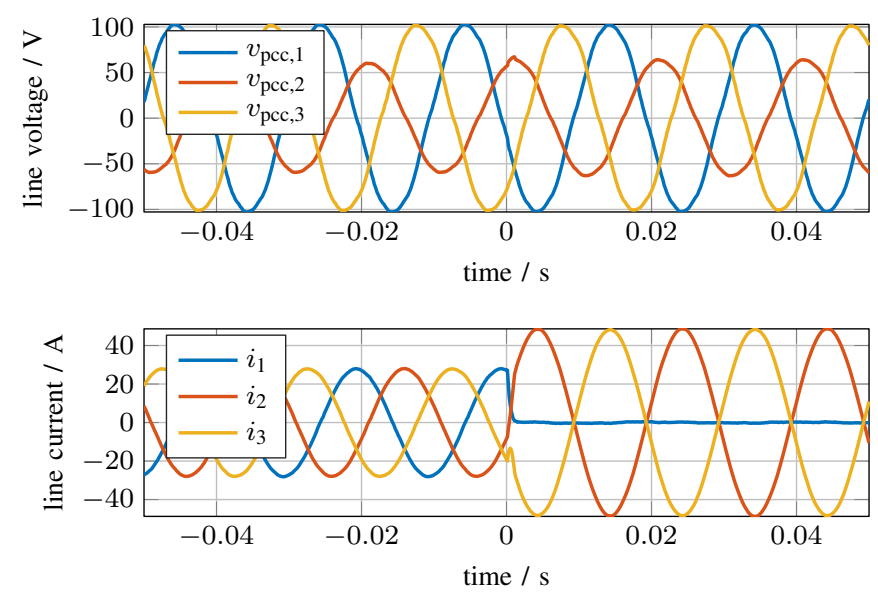

Figure 7. Injection of positive and negative sequence currents

Up to $t=0 \mathrm{~s}$ only a positive sequence grid current was defined as reference value with $i_{\text {active }}^{*}=0 \mathrm{~A}$ and $i_{\text {reactive }}^{*}=$ $-\sqrt{2} \cdot 20 \mathrm{~A}$. At $t=0 \mathrm{~s}$ a negative sequence current reference of $i_{\text {neg,real }}^{*}=0 \mathrm{~A}$ and $i_{\text {neg,imag }}^{*}=-\sqrt{2} \cdot 20 \mathrm{~A}$ was added.

With the implemented voltage prediction algorithm described in Section 5 the unsymmetrical grid voltage does not impose any problems for the control and the current references are met with high accuracy.

\section{CONCLUSION}

The proposed control scheme enables high quality current control even under severe grid conditions. With the usage of iron powder inductances a high overload capability of up to $250 \%$ of the rated current was realized with the laboratory prototype. Like shown, the model for control implementation was given in most general form, so the control scheme is also adaptable to three-phase inductors.

Additionally, the control scheme features a freely programmable current trajectory, enabling advanced ancillary services for power converters, which will be evaluated in future work.

\section{ACKNOWLEDGMENT}

We gratefully acknowledge the financial support from the German Federal Ministry for Economic Affairs and Energy (BMWi) for the project Advanced Decentral Grid Control (funding number "03ET7539F") within the initiative "Zukunftsfähige Stromnetze".

\section{REFERENCES}

[1] Federal Ministry of Economics and Technology (BMWi) and Federal Ministry for the Environment, Nature Conservation and Nuclear Safety (BMU), "First Monitoring Report Energy of the future," 2012.

[2] G. Lammert et al., "Dynamic grid support in low voltage grids - fault ride-through and reactive power/voltage support during grid disturbances," in Power Systems Computation Conference (PSCC). IEEE, Aug 2014, pp. 1-7. [Online]. Available: http: //dx.doi.org/10.1109/PSCC.2014.7038468

[3] C. H. Benz, W.-T. Franke, and F. W. Fuchs, "Low voltage ride through capability of a $5 \mathrm{~kW}$ grid-tied solar inverter," in Power Electronics and Motion Control Conference (EPE/PEMC). IEEE, Sep 2010. [Online]. Available: http://dx.doi.org/10.1109/EPEPEMC.2010.5606829

[4] G. M. S. Azevedo et al., "Photovoltaic inverters with fault ridethrough Capability," 2009 IEEE International Symposium on Industrial Electronics, Jul 2009. [Online]. Available: http://dx.doi.org/10.1109/ ISIE.2009.5213298

[5] L. Hadjidemetriou, E. Kyriakides, and F. Blaabjerg, "A grid side converter current controller for accurate current injection under normal and fault ride through operation," in 39th Annual Conference of the IEEE Industrial Electronics Society. IEEE, Nov 2013. [Online]. Available: http://dx.doi.org/10.1109/IECON.2013.6699347

[6] A. Hoke et al., "Sizing SiC storage inverters for fast grid frequency support," in 3rd Workshop on Wide Bandgap Power Devices and Applications (WiPDA). IEEE, Nov 2015. [Online]. Available: http://dx.doi.org/10.1109/WiPDA.2015.7369262

[7] Y. Sugawara et al., "Development of a $100 \mathrm{kVA} \mathrm{SiC}$ inverter with high overload capability of $300 \mathrm{kVA}$," in 21st International Symposium on Power Semiconductor Devices \& IC's. IEEE, Jun 2009. [Online]. Available: http://dx.doi.org/10.1109/ISPSD.2009.5158069

[8] J. Rodriguez et al., "Predictive Current Control of a Voltage Source Inverter," IEEE Transactions on Industrial Electronics, vol. 54, no. 1, pp. 495-503, Feb 2007. [Online]. Available: http://dx.doi.org/10.1109/ TIE.2006.888802

[9] P. Cortes et al., "Predictive Control in Power Electronics and Drives," IEEE Transactions on Industrial Electronics, vol. 55, no. 12, pp. 4312-4324, Dec 2008. [Online]. Available: http: //dx.doi.org/10.1109/TIE.2008.2007480

[10] J. Richter, T. Gemaßmer, and M. Doppelbauer, "Predictive current control of saturated cross-coupled permanent magnet synchronous machines," in Power Electronics, Electrical Drives, Automation and Motion (SPEEDAM). IEEE, Jun 2014. [Online]. Available: http://dx.doi.org/10.1109/SPEEDAM.2014.6871930

[11] D. Yazdani, A. Bakhshai, and P. Jain, "Grid synchronization techniques for converter interfaced distributed generation systems," in Energy Conversion Congress and Exposition. IEEE, Sep 2009. [Online]. Available: http://dx.doi.org/10.1109/ECCE.2009.5316522

[12] F. D. Freijedo et al., "Grid-synchronization methods for power converters," in 35th Annual Conference of IEEE Industrial Electronics Society. Institute of Electrical \& Electronics Engineers (IEEE), Nov 2009. [Online]. Available: http://dx.doi.org/10.1109/IECON.2009. 5414976

[13] S. Golestan et al., "Moving Average Filter Based Phase-Locked Loops: Performance Analysis and Design Guidelines," IEEE Transactions on Power Electronics, vol. 29, no. 6, pp. 2750-2763, Jun 2014. [Online]. Available: http://dx.doi.org/10.1109/TPEL.2013.2273461

[14] J. Richter et al., "Mitigation of Current Harmonics in Inverter-Fed Permanent Magnet Synchronous Machines with Nonlinear Magnetics," in Proceedings of PCIM Europe 2015, 2015.

[15] T. Gemaßmer et al., "High Dynamic Rotor Oriented Current Control for Permanent Magnet Synchronous Machines with Saturation Characteristics," in Proceedings of PCIM Europe 2014. VDE, May 2014, pp. $1-8$ 